\title{
A Unique Framework to Describe Short Range Order and Properties of the Ordered State in Alloys
}

\author{
D. Le Bolloc'h*,**, R. Caudron*,**, E. Cabet*, M. Barrachin*,** and A. Finel* \\ * Direction des Matériaux, ONERA, 92322 Châtillon sous Bagneux, France \\ ** Laboratoire Léon Brillouin, CEA/CNRS, 91191 Gif sur Yvette cedex, France
}

\begin{abstract}
Elastic diffuse scattering of neutrons was performed in situ on two different alloys, Ni3 V and $\mathrm{Pt} 3 \mathrm{~V}$. Effective pair interactions of an Ising model were deduced from the measured short-range order parameters in the disordered phase. For this two examples, we obtain potentials that don't depend on temperature. Thus we can predict properties of the ordered state. In Ni3 V, we describe correctly the temperature variation of the dissociation width of superdislocations and we compare the stability of different phases, $\mathrm{Ll}_{2}$ and $\mathrm{DO}_{22}$. In the case of $\mathrm{Pt}_{3} \mathrm{~V}$, we will show how we can relate the measured interactions with the existence of long period structures in the ordered state.
\end{abstract}

\section{INTRODUCTION}

We can describe the ordering energy of a substitutional binary alloy $A_{c} B_{1-c}$ by generalised Ising Hamiltonian,

$$
\mathrm{H}=\sum \mathrm{J}_{\mathrm{nm}}\left(\mathrm{p}_{\mathrm{n}}-\mathrm{c}\right)\left(\mathrm{p}_{\mathrm{m}}-\mathrm{c}\right)+\sum \mathrm{J}_{\mathrm{nml}}\left(\mathrm{p}_{\mathrm{n}}-\mathrm{c}\right)\left(\mathrm{p}_{\mathrm{m}}-\mathrm{c}\right)\left(\mathrm{p}_{1}-\mathrm{c}\right)+\ldots
$$

where $p_{n}$ are occupation numbers taking the values 0 or 1 depending on specie $A$ at site $n$.

This energy represents the difference between the total energy of a given configuration $\left(\mathrm{p}_{\mathrm{n}} \mathrm{p}_{\mathrm{m}} \ldots\right)$ and the energy of the totally disordered alloy.

Many theoretical studies[1-2] have shown that, for transition alloys, the pair interactions are dominant versus multiplet interactions i.e. the internal energy of our system can be written (in a grand-canonical approach) :

$$
\mathrm{H}=\sum \mathrm{V}_{\mathrm{nm}} \sigma_{\mathrm{m}} \sigma_{\mathrm{n}}+\mathrm{h} \sum \sigma_{\mathrm{n}}
$$

where the $\sigma_{n}$ 's, related to the $p_{n}$ 's by $p_{n}=\left(1-\sigma_{n}\right) / 2$, are pseudo spin variables. $\sigma_{n}$ equals +1 or -1 depending whether or not site $\mathrm{n}$ is occupied by an A specie. The V's are the corresponding effective pairwise interactions and $h$ is the chemical potential difference.

In this framework, phase diagrams of our compounds should be deductible from these interactions, along with other properties of ordered state.

The short range order parameters (SRO) $\alpha(\mathbf{R})=\alpha_{\mathrm{mn}}=\left(<\sigma_{m} \sigma_{\mathrm{n}}>-<\sigma_{m}><\sigma_{\mathrm{n}}>\right) / 4 c(1-c)$ with $\mathbf{R}=\mathbf{R}_{\mathrm{n}}-\mathbf{R}_{\mathrm{m}}$, have been determined in situ above the transition temperature, and the pair interactions have been deduced from these data by two different methods: inverse Monte Carlo simulation and inverse cluster variation method (CVM)

\section{EXPERIMENTAL}

The diffuse neutron scattering experiments were performed on the diffuse scattering spectrometer G44 at the laboratoire Leon Brillouin, CEN Saclay, France. We explored two planes of high symmetry, the (001) and (110) planes with an incident wave length $\lambda=2.59 \AA$ (resolution $\Delta \lambda / \lambda \leq 4.10^{-2}$ ). The spectrometer is 
equipped with a vacuum chamber ( $10^{-6}$ torr) and a furnace which surrounds the sample and enable us to reach $1300 \mathrm{C}^{\circ}$. At temperatures corresponding to the disordered state of our samples, strong phonon annihilation processes occur, and an energy analysis is necessary to reject the corresponding intensity. For this, the spectrometer is equipped with a chopper and a time of flight analysis, which allow us to reject inelastic scattering processes with an energy resolution of 5 and $3 \mathrm{meV}$ for phonon annihilation and creation, respectively[3]. In these conditions, we obtain the elastic diffuse scattering for $0.5 \leq q=4 \pi \sin$ $\theta / \lambda \leq 4.5 \AA^{-1}$ with steps $2.5^{\circ}$ in $2 \theta$ and $3^{\circ}$ in $\omega$.

\section{TO OBTAIN THE LOCAL ORDER PARAMETERS}

The general expression for the intensity scattered by a binary alloy at the scattering vector $\mathbf{q}$ is :

$$
\mathrm{I}(\mathbf{q})=\sum_{i=1}^{N} \sum_{j=1}^{N} \mathrm{~b}_{\mathrm{i}} \mathrm{b}_{\mathbf{j}} \exp \left[\mathbf{i} \mathbf{q}\left(\mathbf{R}_{\mathbf{i}}-\mathbf{R}_{\mathrm{j}}+\mathbf{u}_{\mathrm{i}}-\mathbf{u}_{\mathrm{j}}\right]\right.
$$

Where $\mathbf{R}_{\mathbf{i}}$ is the ith lattice position, $b_{\mathbf{i}}$ and $b_{j}$ are the scattering factors for the atom $i$ and $j, \mathbf{u}_{i}$ and $\mathbf{u}_{j}$ are the displacement vectors from the lattice position to the true atom position. The sums run on sites of the FCC lattice (structure of the two alloys studied, $\mathrm{Ni}_{3} \mathrm{~V}$ and $\mathrm{Pt}_{3} \mathrm{~V}$ ). Up to now, our statistical methods don't enable us to deal with combined effects of chemical order and displacements. Thus, it is necessary to obtain the contribution of the local order only: If we consider displacements $\mathbf{u}_{\mathrm{ij}}=\mathbf{u}_{\mathrm{i}}-\mathbf{u}_{\mathrm{j}}$ small compared to the vectors $\mathbf{R}_{\mathbf{j i}}=\mathbf{R}_{\mathbf{i}}-\mathbf{R} \mathbf{j}$, then the exponential can be expanded, and the intensity can be written as a sum of two terms : the first one is the SRO contribution, periodic in the reciprocal lattice. The second one is the contribution of the static atomic displacements, whose effects are more important far away from the origine of the reciprocal lattice. In $\mathrm{Pt}_{3} \mathrm{~V}$, this last contribution seems to be more important than in $\mathrm{Ni}_{3} \mathrm{~V}$ and the exponential is expanded up to the second order :

$$
\exp \left[\mathbf{i q}\left(\mathbf{R}_{\mathrm{ij}}+\mathbf{u}_{\mathrm{ij}}\right)\right] \approx \exp \left[\mathbf{i q} \mathbf{R}_{\mathrm{ij}}\right]\left[1+\mathrm{i}\left(\mathbf{q u} \mathbf{u}_{\mathrm{ij}}\right)-1 / 2\left(\mathbf{q u} \mathbf{u}_{\mathrm{ij}}\right)^{2}\right]
$$

After separation of the Bragg scattering and using thermodynamic averaging, the contribution due solely to the SRO can be written:

$$
\begin{gathered}
\mathrm{I}(\mathbf{q})=\mathrm{I}_{\text {laue }} \cdot \alpha(\mathbf{q}) \\
\alpha(\mathbf{q})=\sum \exp (\mathrm{iq} \mathbf{R}) \alpha(\mathbf{R})
\end{gathered}
$$

where the sum runs on the lattice vectors $\mathbf{R}$ with respect to some origin o. This contribution is just a modulation of the Laue signal intensity $\left(I_{l a u e}=4 \pi c(1-c)\left(b_{a}-b_{b}\right)^{2}\right)$, and is linear in the $\alpha(\mathbf{R})$ 's. From the expression of the intensity, the different parameters characterising the contributions of the local order and distortion can be fitted using a multilinear squares routine based on the singular value decomposition[4].

\section{DETERMINATION OF EFFECTIVE POTENTIALS}

Generally, statistical physics methods are devised in order to solve approximately the Ising model (1) with a given set of potentials $\mathrm{V}$, and to compute the properties of the system, including the SRO parameters $\alpha(\mathbf{R})$. Such procedure can be termed direct. Deducing potentials from SRO requires to solve the inverse problem, and we must employ a trial and error method using two statistical physic approaches.

In a first investigation, an inverse cluster variation method (CVM) limited to the four potential has been used. The CVM is a deterministic method which consists in approximating the probability to obtain a given configuration $\Omega$, by a probability $\rho_{\text {cvm }}\left(\beta_{M}\right)$ associated to a basic cluster $\beta_{M}$. For a given choice of $\beta_{M}$, 
the approximate probability reads:

$$
\rho_{\mathrm{cvm}}=\prod_{\gamma}^{*} \rho_{\gamma}^{\mathrm{a} \gamma} \quad \underset{\beta \gamma ; \beta \supset \gamma}{\sum_{\beta ; \beta}^{*}(-1)^{|\gamma-| \beta \mid}}
$$

where the asterisk means that the product runs over the subcluster of $\gamma$ included in at least one cluster of type $\beta_{\mathrm{M}}$. Actually, this approximation consists in replacing the exact entropy by a linear combination of entropies of finite clusters included in the basic cluster. In the present case, we want to consider pair interactions up to the 4th neighbours in a FCC structure. The smallest cluster we must use in such a case is the so called 13-14 point approximation [5].

In order to investigate longer-range interactions, we had to use an inverse Monte-carlo code. Fundamentally different from the CVM, the Monte-carlo approach is a numerical simulation, free from approximation but subjected to fluctuation and very expensive in terms of calculation time. Now, we will show how various physical properties of the ordered state, can be predicted from potentials obtained in the disordered state.

\section{APPLICATIONS}

For $\mathrm{Ni}_{3} \mathrm{~V}$ [4], which orders at $840^{\circ} \mathrm{C}$, the experiments were performed at $1100^{\circ} \mathrm{C}$. The experimental maps with the SRO contribution only are shown on the figure 1 .

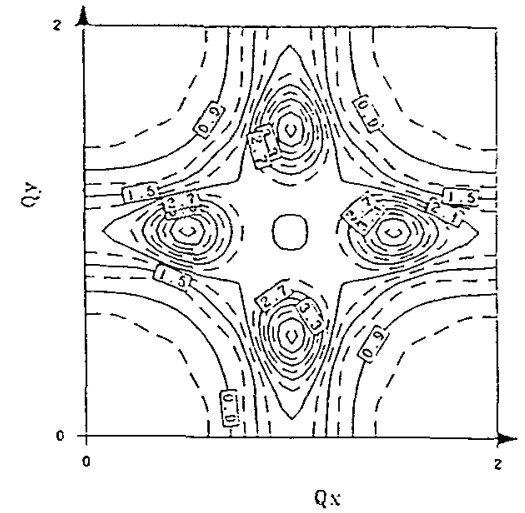

figure 1.a: Experimental diffuse intensity $\alpha(\mathrm{q})$ in $\mathrm{Ni}_{3} \mathrm{~V}$ at $\mathrm{T}=1100^{\circ} \mathrm{C}$ in the (100) plane (Laue units)

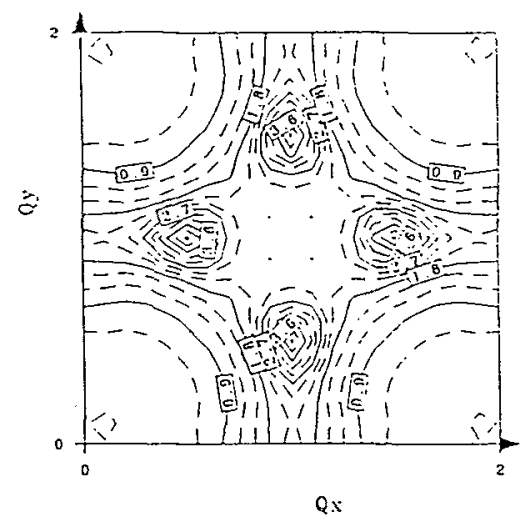

figure 1.b: Calculated diffuse intensity $\alpha(q)$ in the (100) plane, using the optimal interaction set (Laue units)

The inverse MC yielded potentials up to 9th neighbours, but we kept only the best set displayed in the table I. As a check, these interactions were introduced in a Monte Carlo simulation and reproduced closely the experimental map, especially the amplitudes and the shape of the intensity maxima (fig 1b).

\begin{tabular}{|c|c|c|c|c|c|c|c|}
\hline Ni3V & $V_{1}$ & $v_{2}$ & $v_{3}$ & $V_{4}$ & $V_{7}$ & $V_{8}$ & $V_{9}$ \\
\hline$M-C(\mathrm{meV})$ & 36.2 & -7.8 & -0.5 & 3.5 & -0.5 & -2.4 & -1.5 \\
\hline
\end{tabular}

Table L. Effective pair interactions in $\mathrm{Ni}$ V, (meV) 
These effective potentials can be used to predict physical properties: First, by Monte-carlo simulations, we have calculated the transition temperature $T_{c}$ between the disordered phase and the $\mathrm{DO}_{22}$ compound, which is the stable low temperature phase of $\mathrm{Ni}_{3} \mathrm{~V}$. They are in reasonable agreement with the experimental $\mathrm{T}_{\mathrm{C}}$ $\left(\mathrm{T}_{\mathrm{C}}{ }^{\exp }=1045^{\circ} \mathrm{C} ; \mathrm{T}_{\mathrm{C}}{ }^{\mathrm{cal}}=840^{\circ} \mathrm{C}\right)$. The energy difference between the two phases susceptible of being stabilized at low temperature for this stochiometry (the $\mathrm{DO}_{22}$ and the $\mathrm{Ll}_{2}$ phases) has been calculated:

$$
\Delta E=e\left(D_{22}\right)-e\left(L_{2}\right)=-V_{2}+4 V_{3}-4 V_{4}-4 V_{6}+8 V_{7}=-12 \mathrm{meV}
$$

This result is in agreement with the observed stability of $\mathrm{DO}_{22}$ and interesting comparisons have been made with electronic structure calculations[6]: the energy difference between these two phases is eight times lower than the one found by ab initio calculations at $0 \mathrm{~K}$. This discrepancy is partially explained by the electronic excitations.

By transmission electron microscopy (weak beam technique), the dissociation width of dislocations have been observed in the $\mathrm{DO}_{22}$ phase[7]. From these observations, the authors have determined antiphase free energies. A stringent test of the transferability of our set of potentials is to use them to compute antiphase free energies in the ordered state and to compare the computed values to those obtained experimentally. With our Ising model up to the 9th interaction, the excess energies of the conservative antiphase boundaries in the (100) and (111) planes of the $\mathrm{DO}_{22}$ phase are given at zero temperature by :

$$
\begin{gathered}
\xi(100)=2 V_{2}-8 V_{3}+8 V_{4}+8 V_{6}-16 V_{7}-4 V_{8} \\
\xi(111)=V 1-V_{2}-4 V_{3}+2 V_{4}+6 V_{5}+6 V_{6}-12 V_{7}-6 V_{8}+3 V_{9}
\end{gathered}
$$

The excess antiphase free energies at finite temperature are calculated by Monte Carlo simulations. More precisely, using anti-boundary conditions, we first introduce one antiphase into an otherwise homogenous ordered phase and we compute the free energy an a function of temperature (by thermodynamic integration). The antiphase free energy is given by the difference between this free energy and that of the ordered phase without antiphase. The results are shown in figure 2 , along with the experimental data. The agreement is very good.
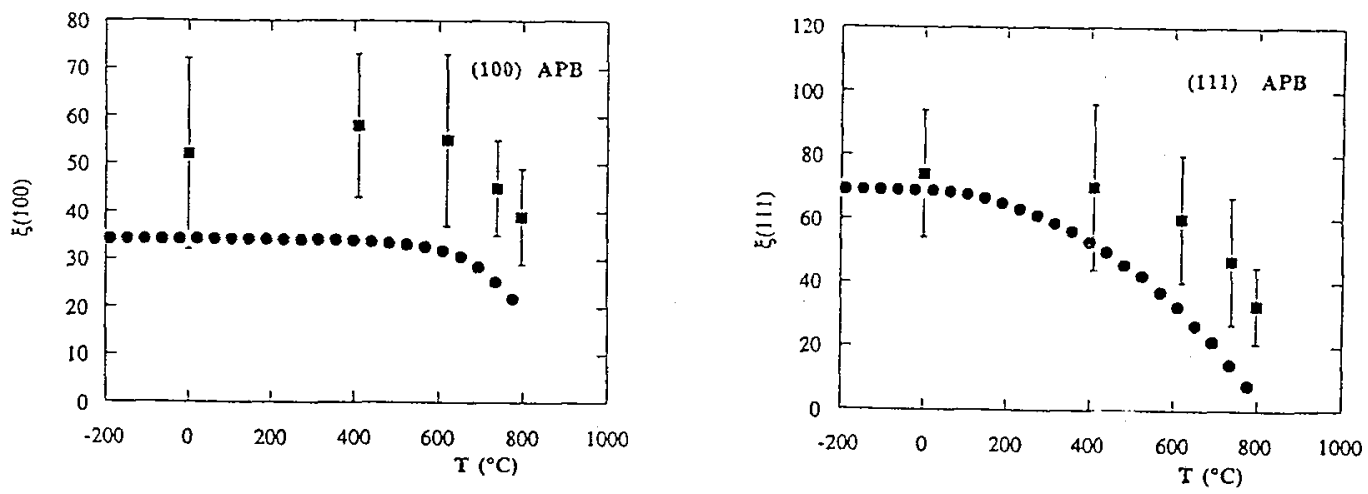

Figure 2: Free energies of the (100) and (111) antiphases in the $\mathrm{DO}_{22}$ phase, as functions of temperature. The circles represent the Monte Carlo calculation using our interaction set. The squares are the experimental data with their errors bars (energy units: meV/atom).

$\mathrm{Pt}_{3} \mathrm{~V}$ alloy, which as $\mathrm{Ni}_{3} \mathrm{~V}$, orders at low temperature on the FCC lattice, has the particular feature of exhibiting different types of order in the ordered state. Electron microscopy observations[8] have shown the presence of long period structures derived from the $\mathrm{L}_{2}$ phase and characterised by the mean distance $\mathrm{M}$ between antiphase boundary along the (100) direction: the $\mathrm{DO}_{22}$ phase between 0 and $900{ }^{\circ} \mathrm{C}$ and the $\mathrm{M}=4 / 3$ phase (three antiphases on four cubes) up to the transition temperature $\mathrm{Tc}=1040^{\circ} \mathrm{C}$. 


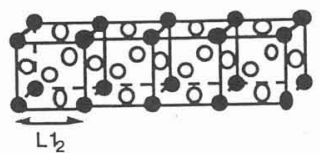

a)

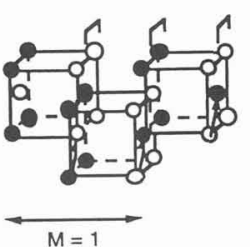

b)

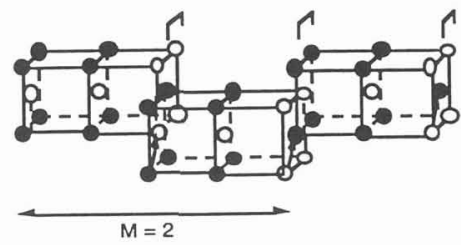

c)

Figure 3: Structures $\mathrm{Ll}_{2}$ (a), $\mathrm{DO}_{22}(\mathrm{M}=1)(\mathrm{b})$, and $\mathrm{M}=2$ (c). The two last structures $\left(\mathrm{DO}_{22}\right.$ and $\mathrm{M}=2$ ) can be described from the basic structure $\mathrm{L}_{2}$ by introducing a modulation of antiphase boundaries (APB).

These configurations can also be modified by varying slightly the electronic structure of the alloy[8]. Such a situation is typical of a quasi-degenerate case, where the energy differences between each phase in competition in the ordered state are very low.

The local order parameters have been obtained from the experiment, using the expansion up to the second order in the distortions (eq. 2). This fit enables us to obtain a better reproduction of the experimental intensity throughout in the reciprocal lattice. The (100) map of SRO measured at $1120^{\circ} \mathrm{C}$ is displayed in fig 4 a.

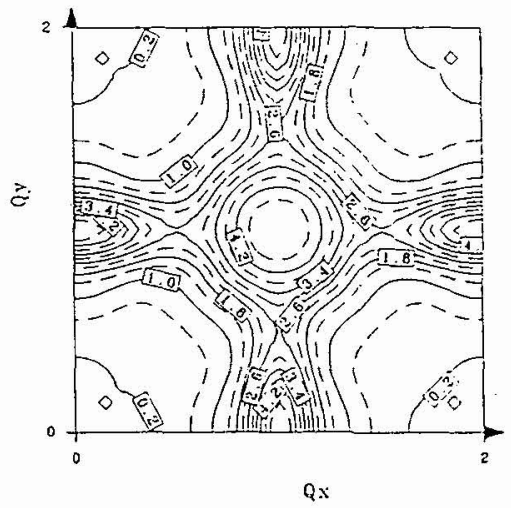

figure 4.a: Experimental diffuse intensity $\alpha(q)$ in $P t_{3} V$ at $\mathrm{T}=1120^{\circ} \mathrm{C}$ in the (100) plane ( Laue units)

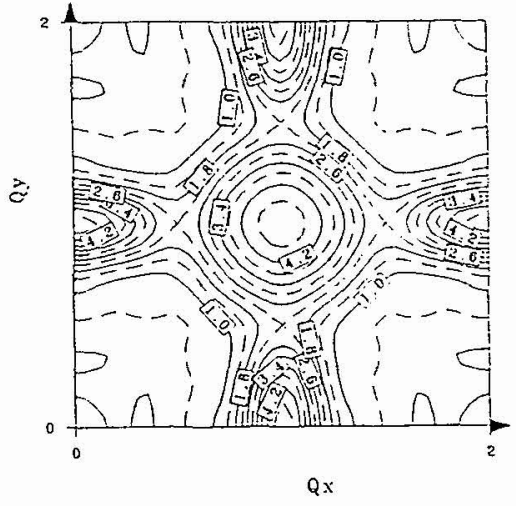

figure 4.b: Calculated diffuse intensity $\alpha(q)$ in the (100) plane, using the optimal interaction set (Laue units)

As a first approximation, we can estimate the parameters of interest from two points of the experiment map. Indeed, if we consider an Hamiltonian of nine interactions, the Clapp and Moss formula gives us a direct relation between the local order parameters and $\Delta \mathrm{E}$ :

$$
\alpha^{-1}(100)-\alpha^{-1}(11 / 20)=-16 c(1-c) \beta \Delta \mathrm{E}
$$

i.e. $\Delta E=+4 \mathrm{meV}$. So, even before any detailed calculation, a direct observation of the map shows us that, in this alloy, the energy difference between $\mathrm{DO}_{22}$ and $\mathrm{L}_{2}$ phases is very low.

This conclusion is confirmed by the results of CVM and inverse Monte Carlo simulation. The two sets of potentials we have obtained are displayed in the table II. 


\begin{tabular}{|c|c|c|c|c|c|c|c|c|c|}
\hline$P t 3 V$ & $V_{1}$ & $V_{2}$ & $V_{3}$ & $V_{4}$ & $V_{5}$ & $V_{6}$ & $V 7$ & $V_{8}$ & $V 9$ \\
\hline$M-C(m e V)$ & 52.8 & -11 & 0.3 & 0.7 & -1.3 & -2.3 & -2.3 & 1.2 & -3.9 \\
\hline$C V M(m e V)$ & 61.5 & -2.25 & 5 & 5 & & & & & \\
\hline
\end{tabular}

Table IX: Effective pair interactions in $\mathrm{Pt} 3 \mathrm{~V}$.

With the set of 9 potentials, we can reproduce accurately the experimental map (fig $4 \mathrm{~b}$ ) and simulate a transition temperature $\left(\mathrm{T}_{\mathrm{C}}{ }^{\mathrm{exp}}=1090^{\circ} \mathrm{C} ; \mathrm{T}_{\mathrm{C}}{ }^{\mathrm{cal}}=830^{\circ} \mathrm{C}\right)$. Despite the high sensitivity of potentials to the data reduction methods, the linear combination of potentials of interest, $\Delta \mathrm{E}$, is stable: $\Delta \mathrm{E}=+0.2 \mathrm{meV}$. Thus, our analysis shows that the energy difference between $D_{22}$ and $L_{1}$ phases is very low. This result explains why long period structures are observed.

\section{CONCLUSION}

In this paper, we explain how we can deduce effective pair interactions of a Ising Model from diffuse scattering of neutrons in the disordered state of two binary alloys $\left(\mathrm{Pt}_{3} \mathrm{~V}\right.$ and $\left.\mathrm{Ni}_{3} \mathrm{~V}\right)$, . With these potentials, we have shown how we can describe not only the disordered state, but also properties of the ground state.

In $\mathrm{Ni}_{3} \mathrm{~V}$, an Ising Hamiltonian expanded up to the 9 th pair interaction, enables us to describe faithfully the experimental map and to simulate accurately the order-disorder transition temperature, the energy difference between $\mathrm{DO}_{22}$ and $\mathrm{Ll}_{2}$ phases, and the evolution of free energies of the (100) and (111) antiphases in the $\mathrm{DO}_{22}$ phase as a function of temperature.

In order to explain a quasi-degenerate case as $\mathrm{P}_{3} \mathrm{~V}$, an analysis with a Hamiltonian with relatively far pair interactions, at least to the 8th neighbour, shows us how small is the difference between two different configurations $\left(\mathrm{DO}_{22}\right.$ and $\left.\mathrm{L}_{2}\right)$. This result is effectively in favour of the stabilization of long period structures in the ground state.

\section{Acknowledgements}

The authors are grateful to D.Regen (ONERA Paris) for the growth of high quality single crystals.

\section{References}

[1] Ducastelle F. and Gautier,J. phys. F6 (1976) 2039

[3] Caudron R.,Sarfati M.,Barrachin M.,Finel A.,Ducastelle F.,F. Solal,J.phys. I 2(1992) 1145.

[4] Barrachin M.,Ph.D.Paris XI-Orsay, 1993.

[5] Finel A.,Ph.D.Paris VI,1987.

[6] Barrachin M.,Finel A.,Caudron R., Pasturel A.,François A.,Phys. Rev. B 50,(1994) 12980.

[7] François A.,Ph.D.Paris VI, 1992.

[8] Cabet E.,Loiseau A.,J. Phys. (France) IV colloque C7,supp. au J. d Phys III 3(1993) 2051.

[2] Ducastelle F., Order and phase stability in alloys, edited by F. R. de Boer and D. G. Pettifor, Cohesion and Structure Vol 3 (North -Holland, Amsterdam,1991). 